\title{
ORTHOPAEDIC ASPECTS OF RHEUMATOID ARTHRITIS
}

BY

\section{JOHN BASTOW}

Attainment of an improved bodily posture is of the utmost importance if the general resistance of the individual to the infection is to be upgraded. The typical posture of rheumatoid arthritis is one of fatigue, with " poking chin," drooping shoulders, flat chest with poor respiratory excursion, visceroptosis, sway-back from the waist, flexed knees, and pronated feet. The key-note of the corrective process is rest and remedial exercises-done at first in recumbency to eliminate the effects of gravity. Abdominal breathing, and pelvic tilting with gluteal and quadriceps contractions, can be practised by the most toxic patient, and as the general condition improves postural training in correct sitting, standing, and walking attitudes should be given. A firm mattress is a sine qua non. The interiorspring mattress and the feather bed should be firmly condemned.

With local articular lesions in the acute stage the most important single aim is the prevention of deformity and the provision of rest by local splintage. The only satisfactory splintage is " tailormade " on the patient, and this, in most cases, is provided by light plaster of Paris casts. Castex splints and easily moulded light aluminium splints are successfully used, and there is an increasing use of plastics, such as perspex, but at present these need to be made on a plaster cast. Particularly insidious early deformities to be watched for are: (a) ulnar deviation of the fingers at the metacarpophalageal joints; (b) flexion contracture of the wrists; (c) adduction contractures of the shoulders; (d) backward and outward subluxation of the knees; and $(e)$ pronation of the feet.

Hands.-Hands are best dealt with by: (1) Rest plasters worn at night to afford complete support to the fingers and wrists; and (2) short cock-up plasters by day, which allow free use of the fingers and thumb and so do not make the patients helpless.

Shoulders.-Shoulders are difficult to splint satisfactorily, and in the acute phase there is no substitute for the abduction splint, usually of well-padded Cramer wire or duralumin.

Knees.-Knees are particularly suitable for plaster correction, and the plasters should always be completeextending from toes to groin. In the knee early flexion contracture has an especially vicious effect by increasing the weight-bearing strain on the articular cartilage, owing to the increased leverage when the patient is standing up.

There are three stages of flexion spasm of a joint which is the site of acute arthritis: (1) where there is flexion spasm which is abolished by rest; (2) where there is flexion spasm abolished by anaesthesia; and (3) flexion deformity, where secondary contracture of muscles and ligaments has occurred and correction cannot be obtained by rest and anaesthesia alone.

In the subacute stage there are: (1) some degree of deformity that requires correction; and (2) danger of relapse or insidious onset of further deformity if corrective splintage is not instituted.

In the chronic stage, with established deformity, the corrective measures necessary often include operation, such as arthroplasty or arthrodesis.

\section{Principles of Splintage}

A. Rest combined with daily exercises to prevent development of adhesions, which are prone to occur early in this disease; ideally, $23 \frac{1}{2}$ hours splintage, with half an hour's exercise during day.

B. Rest must take place in the optimum position for function.

C. If deformity exists it must be corrected so far as is compatible with the degree of tissue damage, either by: (a) anaesthesia only, followed by plasters; (b) serial plasters; or (c) manipulation under anaesthesia, and plasters.

Fallacies.-." Too acute for splintage." No case is too acute for splintage. The more active the joint inflammation the greater need for the rest and relief from spasm that splintage ensures.

"Do not manipulate for fear of flaring up inflammation." There is no need to fear any flare-up of inflammation provided manipulation is followed by splintage and too much correction is not attempted at one sitting.

" Joints will get stiff if rested or splinted; keep going at all costs." This is not true. Joints, if splinted, will not stiffen provided they are allowed one period of movement daily with non-weight-bearing exercise. The quickest way to destroy, and consequently stiffen, a joint is to exercise it excessively when it is hot and swollen.

"Manipulation will increase the range of movement." This is not necessarily so in rheumatoid arthritis. If a patient has knees flexed habitually to 90 degrees with a further range of active flexion to 60 degrees (i.e. 30 degrees range in all), then if you straighten these knees by serial manipulations and plasters until they are extended to 180 degrees, you will find the active range of flexion still 30 degrees, i.e. 180 degrees to 150 degrees. For this reason you must be careful in selecting your cases for manipulation, and not transform a comfortably seated cripple to an uncomfortable standing one who cannot sit.

\section{Points of Technique}

Plasters applied under anaesthesia without manipulation can be unpadded, smoothly applied 
with slab technique and encircling bandages laid on and never pulled tight. The patient should be given one dose of morphine after the anaesthetic, and then pot. brom. gr. 20 (1.3 g.), aspirin gr. 10 $(0.65 \mathrm{~g}$.$) , four-hourly for forty-eight hours, when$ the plasters are split and the joints given daily physiotherapy.

Serial plasters are applied under the influence of a sedative, without manipulation, and split when dry. The patient wears the plasters continuously except for daily physiotherapy; gain in movement is measured weekly. When progress comes to a standstill, then new serial plasters are applied in the best position obtainable and the routine is repeated. Plasters are usually changed at three-weekly intervals.

In manipulation and plaster under anaesthesia controlled force is used, adhesions are broken down and contracted muscles stretched. One must never do too much at one session.

In the case of the knee a gain of anything from 10 to 30 degrees in extension is aimed at, and then plasters are applied with the pressure points padded with felt and gentle corrective pressure maintained until the plaster sets. These plasters are split in fortyeight hours and the patient is given sedatives regularly. The subsequent routine is the same as for serial plasters without anaesthesia.

When activity ceases and the patient is allowed up some splintage is often necessary, at first to counteract the effects of gravity. Light guarding plasters for the knee, wedged heels and arch supports for the feet, ankle steels and T-straps for the ankle, knee-cages and calipers, etc., all have their uses. Gradually an osteoarthritic phase supervenes, when the joint is creaky but no longer painful, and apparatus can often be discarded but must be reapplied at the first signs of re-activity. Night splints are of great use in this stage, and their use should be continued as long as possible. Finally, rehabilitation should take into account the individual's future mode of life, and he or she should, so far as possible, plan their existence to avoid too heavy a strain on their physically handicapped limbs. The Government's Register of Disabled Persons should be of great assistance in securing for these people priority of suitable light jobs and sheltered occupations. 